\title{
A facile method for bright, colour-tunable light-emitting diodes based on Ga-doped $\mathrm{ZnO}$ nanorods
}

\author{
M. Azizar Rahman, John A. Scott, A. Gentle, Matthew R. Phillips, Cuong Ton-That \\ School of Mathematical and Physical Sciences, University of Technology Sydney, Ultimo, NSW \\ 2007, Australia \\ * Corresponding author. Email: Cuong.Ton-That@uts.edu.au
}

\begin{abstract}
Bottom-up fabrication of nanowire-based devices is highly attractive for oxide photonic devices because of high light extraction efficiency; however, unsatisfactory electrical injection into $\mathrm{ZnO}$ and poor carrier transport properties of nanowires severely limit their practical applications. Here, we demonstrate that $\mathrm{ZnO}$ nanorods doped with Ga donors by in-situ dopant incorporation during vapour-solid growth exhibit superior optoelectronic properties that exceed those currently synthesized by chemical vapour deposition, and accordingly can be electrically integrated into Sibased photonic devices. Significantly, the doping method was found to improve the nanorod quality by decreasing the concentration of point defects. Light emitting diodes (LEDs) fabricated from the Ga-doped $\mathrm{ZnO}$ nanorod/p-Si heterojunction display bright and colour-tunable electroluminescence (EL). These nanorod LEDs possess a dramatically enhanced performance and an order of magnitude higher EL compared with equivalent devices fabricated with undoped nanorods. These results point to an effective route for large-scale fabrication of conductive, single-crystalline $\mathrm{ZnO}$ nanorods for photonic and optoelectronic applications.
\end{abstract}

Keywords: ZnO nanorods; Ga doping; colour-tunable LED; electroluminescence 


\section{Introduction}

Although significant accomplishments have been attained in nitride-based light emitting devices, the pursuit of higher emission intensity and long-life photonic devices never ceases. Compared with thin films, nanowires offer immense potential in photonics as they can support lasing and enhance light extraction efficiency by virtue of nanowire waveguide properties. ${ }^{[1-3]}$ Although Gallium Nitride (GaN) has become a key material for the light emitting diode (LED) technology operating in a wide range of colours, ${ }^{[4]} \mathrm{GaN}$ nanowires are technically difficult to grow, requiring advanced semiconductor growth reactors. Conversely, $\mathrm{ZnO}$ nanowires can be fabricated using the simple vapour transport method. Accordingly, $\mathrm{ZnO}$ nanowires have emerged as one of the most important building block in photonics and have been exploited in LEDs, ${ }^{[5-7]}$ laser $\operatorname{diodes}^{[8,9]}$ and multi-coloured displays. ${ }^{[10]}$ Due to the lack of stable p-type doping in ZnO, most LED devices are developed based on p-n heterojunctions. In general, $\mathrm{ZnO}$ heterojunction diodes exhibit inefficient electrical injection and have weaker light emission than homojunction devices because band offsets at the interface can reduce carrier injection. ${ }^{[6,11]}$ Here, we present a facile route for tailoring the optical and electrical properties of $\mathrm{ZnO}$ nanorods by in-situ $\mathrm{Ga}$ doping. The doped nanorods have significantly improved optoelectronic properties that open the door for the development of durable, high-performance photonic devices.

With a large exciton binding energy of $60 \mathrm{meV}, \mathrm{ZnO}$ nano- and micro-structures have been recognized as competent candidates for the fabrication of high-performance optoelectronic devices. However, $\mathrm{ZnO}$ nanowires have poor carrier transport properties, leading to low carrier injection into $\mathrm{ZnO}$ and weak light output. ${ }^{[6,12,13]}$ These issues arise largely from inherent limitations associated with low carrier concentrations in $\mathrm{ZnO}$ and high energy barrier heights of $\mathrm{ZnO}$ nanowire heterojunctions. ${ }^{[14,15]}$ To overcome this issue, considerable efforts have been dedicated to controlled incorporation of n-type and p-type dopants. Among various n-type dopants, Ga has been recognized as one of the most efficient dopants for enhancing the electrical conductivity of $\mathrm{ZnO}$ 
nanorods. ${ }^{[16,17]}$ The bond length $\mathrm{Ga}-\mathrm{O}(0.192 \mathrm{~nm})$ is almost identical to that of $\mathrm{Zn}-\mathrm{O}(0.197 \mathrm{~nm})$, resulting minimal distortion in the lattice. Taking advantage of the Ga donor's excellent lattice match, band gap engineering has been successfully demonstrated for Ga-doped ZnO nanowires with a maximum red shift of $\sim 0.3 \mathrm{eV}$ in the $\mathrm{UV}$ range. ${ }^{[18]}$ Shen et al. recently reported the observation of electroluminescence (EL) in micrometer-sized Ga-doped $\mathrm{ZnO}$ rods at high temperatures. ${ }^{[19,20]}$ In this work, the authors reported temperature-dependent optical emission in Ga-doped $\mathrm{ZnO}$ rods, which was attributed to the effect of localized Joule heating. The luminescence performance of $\mathrm{p}$-n heterojunction diodes employing Ga-doped $\mathrm{ZnO}$ nanorods has not been investigated thus far. For practical applications, it is important to develop an efficient method to widely tune the emission of $\mathrm{ZnO}$ nanowire heterojunctions in the visible range. In this investigation, vertically oriented Ga-doped $\mathrm{ZnO}$ nanorods were fabricated and applied to $\mathrm{p}$-n heterojunction LEDs. In contrast with previous work, our LED devices employing the p-n heterojunction of Ga-doped nanorods produce chromaticity-stable emission from yellow to red colours without heating effects. Furthermore, compared with equivalent devices made with pristine nanorods, the electrically driven LED devices based on Ga-doped nanorods exhibit a significantly lower turn-on voltage and higher electrical injection, and colour-tunable EL that is an order of magnitude brighter.

\section{Experimental section}

Growth and characterization of Ga-doped $\mathrm{ZnO}$ nanorods: Highly oriented Ga-doped $\mathrm{ZnO}$ nanorods were grown on heavily B doped p-type Si substrates (resistivity 8.7 $\Omega . c m$ and hole concentration $1.44 \times 10^{17} \mathrm{~cm}^{-3}$ ) using a $\mathrm{ZnO}$ seed layer by the vapour solid technique. The $\mathrm{ZnO}$ seed layer was pre-deposited via carbothermal reduction of $\mathrm{ZnO}$ powder as described in our previous work. ${ }^{[21]}$ The Si substrate was cleaned ultrasonically using acetone, isopropanol and deionized water, followed by nitrogen blow-dry. Different Ga concentrations in nanorods were achieved by adding different amounts of $\mathrm{Ga}_{2} \mathrm{O}_{3}$ powder to the source material with concentrations 
up to 10 at $\%$. The source material was heated at $950^{\circ} \mathrm{C}$ under a constant flow of $\mathrm{Ar} / \mathrm{O}_{2}$ gas during growth. The morphological and elemental X-ray microanalyses were performed using Zeiss Supra 55VP scanning electron microscope (SEM) equipped with an Energy Dispersive X-ray (EDX) spectrometer for qualitative elemental mapping. The elemental composition (at \%) of the pristine and Ga-doped ZnO nanorods were determined by Laser Ablation (Teledyne Cetac LSX-266) Inductively Coupled Plasma Mass Spectrometry (Agilent 7500cx) (LA-ICP-MS). Raman spectra of undoped and Ga-doped nanorods were acquired using a LabRAM HR800 spectrometer with the $457 \mathrm{~nm}$ laser excitation line. For TEM imaging, nanorods were removed from the substrate by sonication and drop cast onto a 400 mesh $\mathrm{Cu}$ grid. TEM and Selected-Area Electron Diffraction (SAED) analysis was carried out on a FEI Tecnai T20 TEM.

Device fabrication: After the growth of Ga-doped nanorod arrays (nanorod length $\sim 1 \mu \mathrm{m}$ ), a thick layer of polymethyl methacrylate (PMMA) was carefully deposited by spin coating to fill up the gap among the nanorods. Oxygen plasma (oxygen flow rate of $20 \mathrm{sccm}$ for $20 \mathrm{mins}$ ) was then applied to etch away the 150-nm top section of the PMMA layer, exposing the tips of $\mathrm{ZnO}$ nanorods. The top ITO electrode (200 nm thick) was deposited onto the exposed nanorod tips through a shadow mask using an Edwards EO6 deposition system. Film thickness was measured using a J. A. Woollam spectroscopic ellipsometer and high resolution SEM; the thickness was $\sim 750$ and $200 \mathrm{~nm}$ for the PMMA and ITO layers, respectively. The current-voltage (I-V) characteristics of the devices were measured by using a Yokogawa GS610 source-measure unit. The electroluminescence (EL) and cathodoluminescence (CL) spectra were measured by an Ocean Optics QE Pro spectrometer; all the spectra were corrected for total system response.

\section{Results and discussion}

Fig. 1(a) shows an SEM image of Ga-doped $\mathrm{ZnO}$ nanorods, which display hexagonal end facets and [0001] growth direction, as confirmed also by X-ray Diffraction results (see Supplementary Information Fig. 1S). The nanorods typically have diameters of $\sim 100 \mathrm{~nm}$ and 
lengths of $\sim 1 \mu \mathrm{m}$. The EDX elemental maps [Fig. 1(b)] of a single Ga-doped nanorod extracted from the substrate show a consistent distribution of $\mathrm{Ga}$ and $\mathrm{Zn}$ along its length indicating successful incorporation of high $\mathrm{Ga}$ concentrations up to 1.4 at \% into the nanowires using our transport growth technique. The presence of $\mathrm{Ga}$ and its high uniformity in the length of the nanorods were further confirmed by time-resolved LA-ICP-MS depth profiles, shown in Fig. 2S. The Raman spectra of the undoped and Ga-doped nanowires are presented in Fig. 1(c), which shows typical $\mathrm{ZnO}$ lattice modes at $331 \mathrm{~cm}^{-1}\left(E_{2}^{\text {high }}-E_{2}^{\text {low }}\right)$ and $438 \mathrm{~cm}^{-1}\left(E_{2}^{\text {high }}\right)$. The strain-sensitive Raman $E_{2}^{\text {high }}$ is located at $437.8 \pm 0.2 \mathrm{~cm}^{-1}$ for both undoped and Ga-doped nanorods, indicating a minimal compressive strain due to the incorporation of Ga. The marked increase in the $E_{2}^{\text {high }}$ mode after Ga doping which signifies a significant improvement in optical quality of $\mathrm{ZnO}$ nanorods. ${ }^{[22]}$ The higher crystallinity of the Ga-doped $\mathrm{ZnO}$ nanorods can be attributed to a lower density of point defects as $V_{\mathrm{Zn}}$ defects are filled by substitutional Ga donors, which have almost identical ionic size to the $\mathrm{Zn}^{2+}$ host cations. ${ }^{[23]}$ This is evidenced by the dramatic quenching of the green emission band at $2.4 \mathrm{eV}$, which can be attributed to $V_{\mathrm{Zn}},{ }^{[24]}$ after the Ga doping (see the CL spectra in Supplementary Information Fig. 3S). The emergence of the red emission at $1.8 \mathrm{eV}$ is due to the formation of $\mathrm{Ga}$ defect complexes, in particular $\mathrm{GaZn}_{\mathrm{Z}} \mathrm{O}_{i}$ and $\mathrm{GaZn}-V_{\mathrm{Zn}}$ pairs, as a result of defect compensation. ${ }^{[25,26]}$ The crystal structure and orientation of the nanorods were also confirmed by SAED in a TEM analysis. A typical TEM bright field image and SAED pattern of an isolated Ga-doped $\mathrm{ZnO}$ [0001] nanorod are shown in Fig. 1(d). The dark bands in the TEM image are due to bend contour contrast arising from strain along the length of the Ga-doped $\mathrm{ZnO}$ nanowire while the high quality SAED patterns confirm the single-crystal nature of nanorods. The diffuse streaks seen superimposed on the Bragg diffraction spots that extend in the direction perpendicular to the $g<0001>$ reciprocal lattice vector, shown in the zoomed-in image in the inset, confirms that the nanorod is oriented along the $\mathrm{c}$ axis. This result confirms that the nanorod is oriented along the [0001] direction and that $\mathrm{Ga}$ doping does not affect the usual $c$-axis growth habit of $\mathrm{ZnO}$ nanorods. 

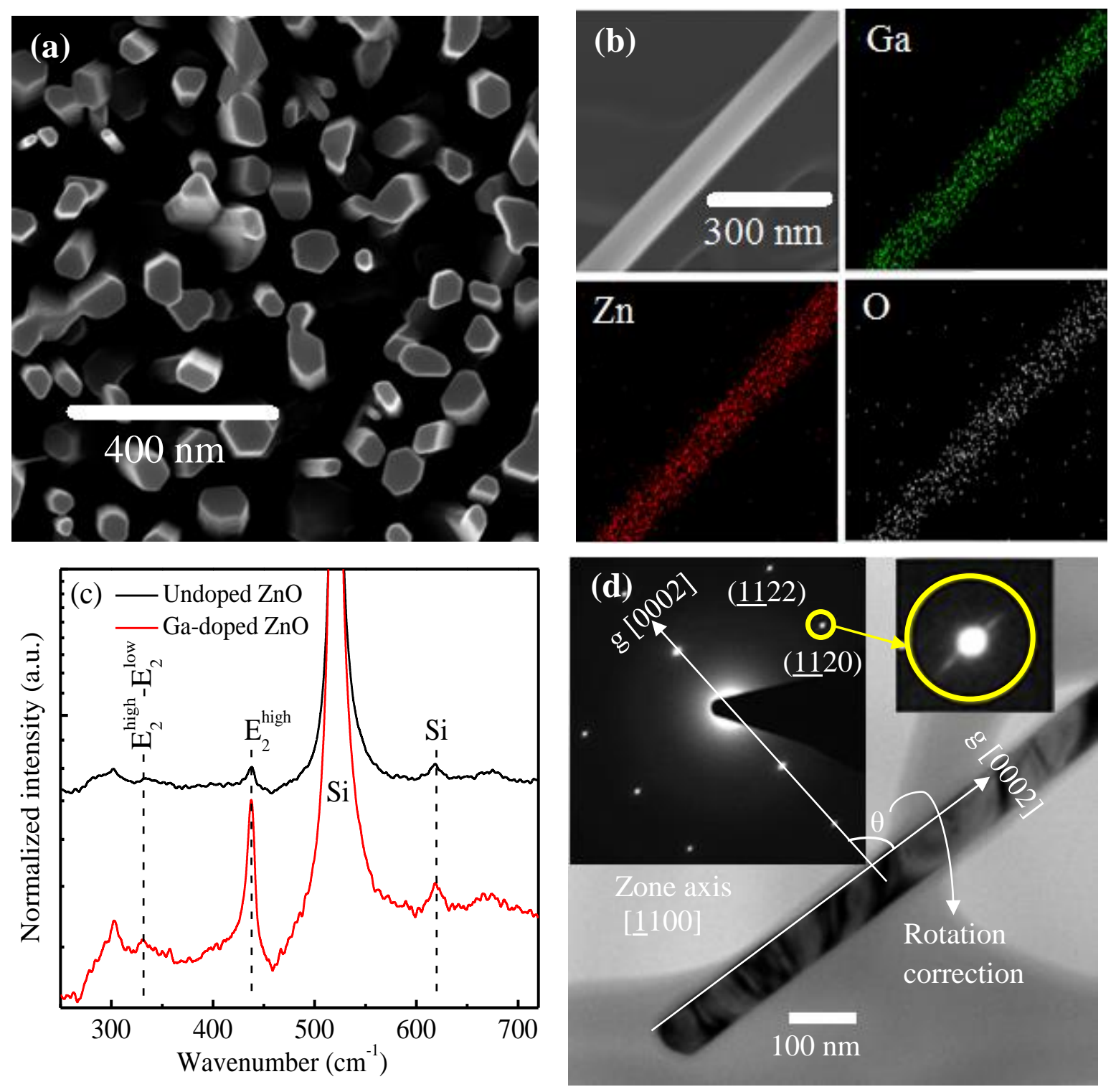

Fig. 1 (a) SEM micrograph of 0.2 at \% Ga-doped $\mathrm{ZnO}$ nanorods grown on $p$-type Si substrate. (b) SEM image $10 \mathrm{kV}$ of an extracted nanorod and corresponding EDX maps of $\mathrm{Zn}, \mathrm{Ga}$ and $\mathrm{O}$ elemental distributions, showing a consistent distribution of the Ga dopant along the length of the nanowire. (c) Comparison of the Raman spectra for undoped and Ga-doped $\mathrm{ZnO}$ nanorods, showing a substantial increase in the $E_{2}^{\text {high }}$ mode after the Ga doping. (d) TEM image $200 \mathrm{kV}$ of a single Ga-doped $\mathrm{ZnO}$ nanorod and its corresponding indexed SAED patterns (inset). The diffraction streaks that extend in the direction perpendicular to the $\mathrm{g}_{[0001]}$ reciprocal lattice vector confirms that the Ga-doped $\mathrm{ZnO}$ nanorod is grown along the $c$-axis orientation.

The $\mathrm{ZnO}$ nanorods/p-Si heterostructure was used to construct a LED as illustrated in the schematic in Fig. 2(a). The tips of the Ga-doped $\mathrm{ZnO}$ nanorods were directly contacted with an 
ITO layer; the p-Si substrate was spaced by a thin PMMA layer (deposited by spin coating) in order to prevent direct contact between the ITO electrode and p-Si substrate. Fig. 2(b) presents the $I-V$ characteristics of nanorods/p-Si heterojunctions measured under direct current biased conditions at room temperature, displaying a characteristic diode behaviour for both undoped and Ga-doped $\mathrm{ZnO}$ nanorods. Under the same bias, the LED devices of the same area fabricated from Ga-doped nanorods exhibit a much higher current density. This suggests that the incorporated Ga atoms form additional midgap states especially $\mathrm{GaZn}-\mathrm{V}_{\mathrm{Zn}}$ pairs, which lower carrier injection barriers for the heterojunction. The turn-on voltage is $4.5 \mathrm{~V}$ for the undoped nanorod LED and monotonically decreases with increasing Ga doping concentration over the range 0-1.4 Ga at\% (see Fig. 4S for the turn-on voltage). The $I-V$ characteristics of the heterojunction diode were further analysed by plotting the data on a log-log scale [Fig. 2(c)]. In region I, $V<3.8 \mathrm{~V}$, the junction current increases almost linearly with applied voltage, $I \propto V^{1.1}$, which demonstrates the carrier transport mechanism followed Ohm's law due to the inter-band electron tunnelling at the Si-ZnO interface. ${ }^{[27]}$ In the region II, $V>3.8 \mathrm{~V}$, the current increases exponentially, $I \propto V^{11.7}$, which is attributed to a recombination-tunnelling mechanism commonly observed in wide band gap semiconductor p-n junctions. ${ }^{[28]}$ 

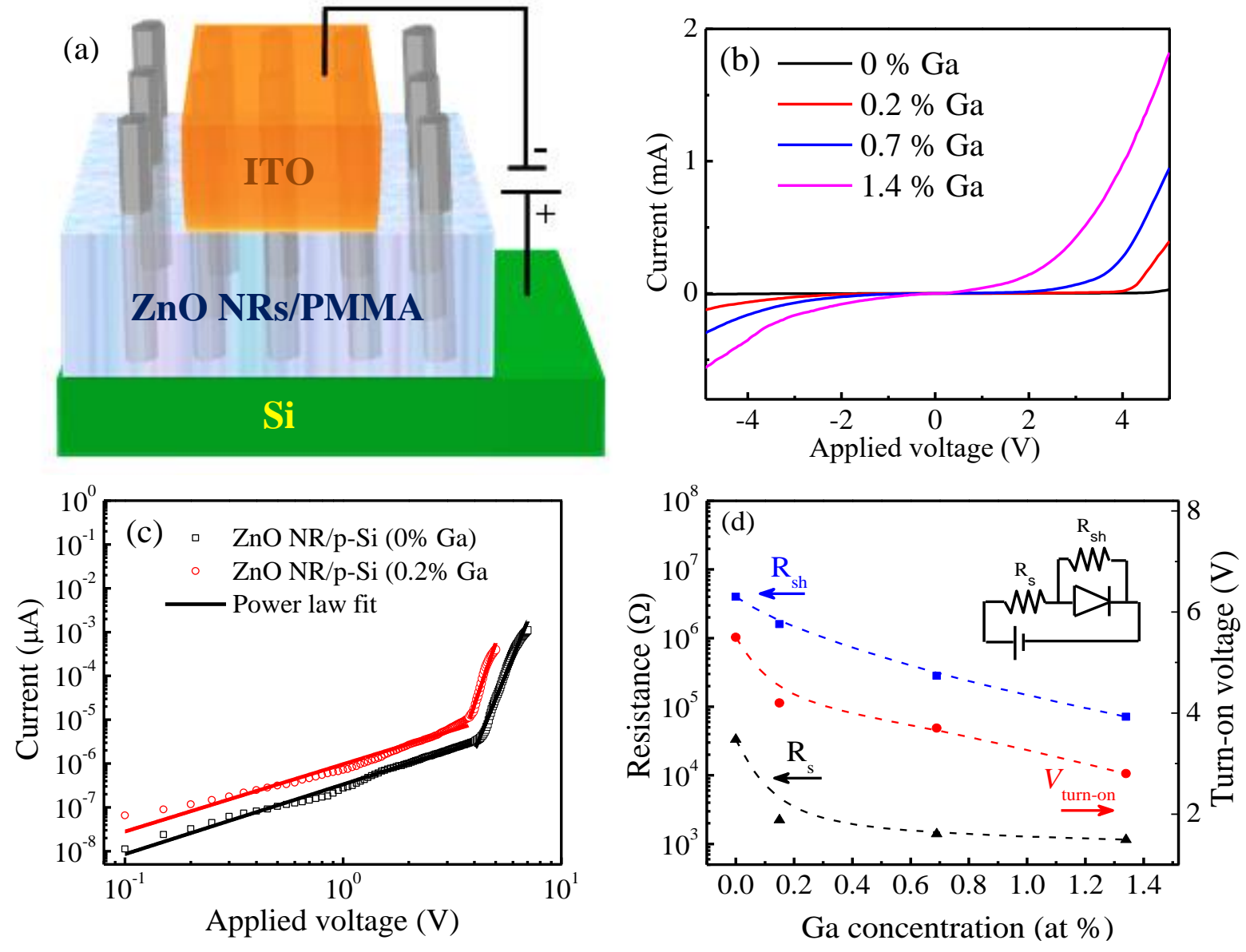

Fig. 2 (a) Schematic illustration of the Ga-doped $\mathrm{ZnO}$ nanorods/p-Si heterojunction LED device structure. (b) $I-V$ characteristic of the ITO/nanorods/p-Si heterojunction LED, showing substantial enhancement of the electrical conduction of the heterojunction with increasing Ga doping concentration. (c) $I-V$ characteristics in the log-log plot curve displaying two regions with different characteristics: $I \propto V^{1.1}$ at $V<3.5 \mathrm{~V}$ and $I \propto V^{11.7}$ at $V>3.5 \mathrm{~V}$. (d) Variations of shunt resistance $\left(R_{\text {sh }}\right)$, series resistance $\left(R_{s}\right)$ and turn-on voltage of the heterojunction as a function of Ga doping concentration. Inset: equivalent circuit used to model the electrical characteristics of the LEDs, consisting of a shunt resistance, $R_{s h}$, and series resistance, $R_{s}$.

The $I-V$ characteristics were analysed by using a one-diode equivalent circuit model, ${ }^{[29]}$ shown in the inset of Fig. 2(d). The total series resistance, $R_{s}$, accounts for Ohmic losses due to the resistance of $\mathrm{Si}$ substrate and $\mathrm{ZnO}$ nanorods, the shunt resistance, $\mathrm{R}_{\mathrm{sh}}$, represents the current leakage across the heterojunction. $R_{\mathrm{sh}}$ and $R_{\mathrm{S}}$ can be determined from the slope of the dark $I-V$ curve at near-zero voltage $(-0.3 \mathrm{~V}<V<0.3 \mathrm{~V})$ and the linear, high current region, respectively (see Fig. $5 \mathrm{~S}$ for an example of $I-V$ curve analysis). ${ }^{[29]}$ The $\mathrm{R}_{\mathrm{s}}$ and $\mathrm{R}_{\mathrm{sh}}$ values of the $\mathrm{ZnO}$ nanorod/Si 
LED are shown in Fig. 2(d), which also displays the turn-on voltage. For the LED fabricated with undoped $\mathrm{ZnO}$ nanorods, $\mathrm{R}_{\mathrm{s}}=34 \pm 2 \mathrm{k} \Omega$, which is large due to the high resistance of the nanorods. The $\mathrm{R}_{\mathrm{s}}$ value decreases dramatically by two orders of magnitude upon the use of Ga-doped $\mathrm{ZnO}$ nanorods in identical LED devices; this can be attributed to the improved nanorod crystal quality and the activation of Gazn shallow donors, which in turn lead to higher conductivity of the nanorods. The reason for the decrease in $\mathrm{R}_{\text {sh }}$ in the LEDs fabricated with Ga-doped $\mathrm{ZnO}$ nanorods is unclear but likely due to increasing current passing along the Ga-doped $\mathrm{ZnO}$ nanorod sidewalls and bypassing the heterojunction. The decrease in the turn-on voltage with increasing Ga doping concentration can be directly attributed to lower total series resistance across the LED device.

EL from the ZnO nanorod/p-Si heterojunction LEDs is observed when the Si side of the hetrostructure is positively biased and was sufficiently intense to be clearly observed by the naked eye at applied bias above $6 \mathrm{~V}$. EL spectra acquired under identical conditions at room temperature, shown in Fig. 3(a), reveal increased EL intensity by an order of magnitude when Ga-doped nanorods are used in the $\mathrm{ZnO} / \mathrm{p}-\mathrm{Si}$ heterojunction LED device. The LED fabricated from undoped $\mathrm{ZnO}$ nanorods exhibits a predominantly $\mathrm{YL}$ EL centered at $2.05 \mathrm{eV}$, which is quenched upon doping with Ga. For Ga-doped nanorods, the LEDs exhibit a strong RL EL band peaking at 1.78 eV. A typical CL spectrum of updoped $\mathrm{ZnO}$ nanorods consist of a weak near-band-edge ultraviolet emission and an intense defect-related green emission band at $2.41 \mathrm{eV}$, which is characteristic of O-rich grown $\mathrm{ZnO}$ nanowires with a high concentration of $V_{\mathrm{Zn}}$ defects. ${ }^{[24]}$ Conversely the EL from LED devices only emits light in the visible range, indicating the EL emission originates from radiative recombination of injected carriers at deep level defects in $\mathrm{ZnO}$ nanorods. The EL integrated intensity and current injection into the LEDs are plotted as a function of applied voltage in Fig. 3(b). The current through the nanorod LED increases remarkably with increasing Ga concentration due to higher conductivity in the Ga-doped nanorods. The EL intensity from a LED device fabricated with 1.4 at $\%$ Ga-doped $\mathrm{ZnO}$ nanorods is higher than the identical device made 
with pristine $\mathrm{ZnO}$ nanorods by one order of magnitude at the same bias voltage. Since the design of the prototype nanorod-based LEDs in this work has not been optimised for lighting applications, their efficiency cannot be competitive compared with those of commercial LED devices. The results here, however, clearly shows that the in-situ Ga doping method can lead to both an enhancement of electroluminescence by an order of magnitude as well as colour tuning capability in $\mathrm{ZnO}$ nanorod-based LEDs.
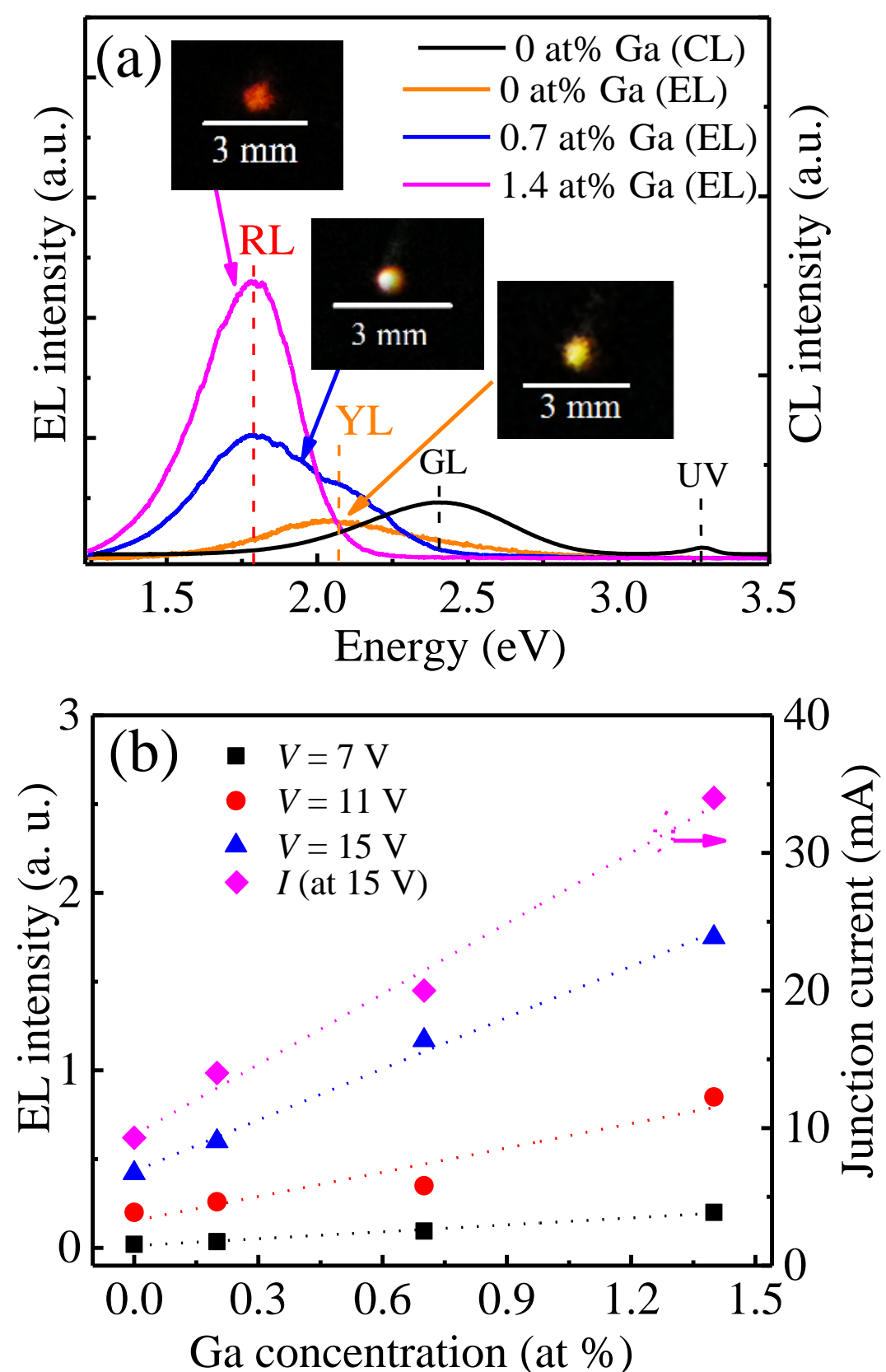

Fig. 3 (a) Room temperature EL spectra of $\mathrm{ZnO}$ nanorod/p-Si LED devices that are fabricated with different Ga doping levels and their corresponding photos of the EL emission. The CL spectrum of 
the undoped $\mathrm{ZnO}$ nanorods is also shown for comparison. The LED devices display two chromaticity-stable emission bands independent of applied voltage (see Supplementary Information Fig. 6S for the EL spectra of the LEDs): yellow luminescence (YL) at $2.05 \mathrm{eV}$ and red luminescence (RL) at $1.78 \mathrm{eV}$. With increasing Ga concentration in the nanorods, the EL is shifted from YL to RL band, and increases dramatically in intensity. (b) EL integrated intensity and junction current as a function of $\mathrm{Ga}$ concentration at different bias voltages.

The observation of the major differences between EL and CL of the Ga-doped nanorods is interesting but complicated. With increasing forward bias voltage, the EL intensity increases without changes in the spectral shape and emission energy (see Supplementary Information Fig. $6 \mathrm{~S}$ for the EL spectra), indicating that the EL arises from the heterojunction without Joule heating effects, allowing for stable and durable nanorod-based LEDs. This is a significant advantage of the direct growth of Ga-doped $\mathrm{ZnO}$ nanorods on $\mathrm{Si}$ (and other substrates that are thermally conductive) as the Si substrate acts as an excellent heat-sink for the nanorod heterojunction, ${ }^{[30]}$ which makes the chromaticity of the LED thermally stable. In nanosized junctions such as in the p-Si/nanorod LED the depletion region is influenced by interface and edge effects. The absence of the GL emission in the EL spectra of the LED can be explained by the fact that $V_{\mathrm{Zn}}$ defects are passivated by forming $\mathrm{GaZn}-\mathrm{V}_{\mathrm{Zn}}$ complexes in highly Ga-doped $\mathrm{ZnO} .{ }^{[26,31]}$ A possible mechanism responsible for the $\mathrm{EL}$ enhancement due to the $\mathrm{Ga}$ doping of the $\mathrm{ZnO}$ nanorods is illustrated in the band alignment diagram of the $\mathrm{p}-\mathrm{Si} / \mathrm{ZnO}$ heterojunction (Fig. 4), derived from the Anderson model. ${ }^{[32]}$ Based on the electron affinities of $\mathrm{Si}\left(\psi_{S i}=4.05 \mathrm{eV}\right)$ and $\mathrm{ZnO}\left(\psi_{\mathrm{ZnO}}=4.35 \mathrm{eV}\right)$, the band offsets are expected to be $\Delta E_{\mathrm{c}} \sim 0.3$ and $\Delta E_{\mathrm{v}} \sim 2.5 \mathrm{eV}$. The large $\Delta E_{\mathrm{v}}$ value means that hole injection into the valence band of undoped $\mathrm{ZnO}$ is strongly suppressed, leading to low light emission in the LED based on undoped nanorod. This problem could be overcome by Ga doping, which results in deep acceptor states, especially $\mathrm{GaZn}_{\mathrm{Zn}}-\mathrm{V}_{\mathrm{Zn}}$ and $\mathrm{GaZn}_{\mathrm{Z}}-\mathrm{O}_{\mathrm{i}}$, as $\mathrm{Ga}$ donors preferentially interact with acceptor-like defects. ${ }^{[26,33]}$ The availability of these defect complexes reduce bias voltage required to inject holes into $\mathrm{ZnO}$ nanorods. Under forward bias, the Si bands are shifted downwards, moving 
the $\mathrm{Si}$ valence band closer to the acceptor states in Ga-doped $\mathrm{ZnO}$. At low bias voltages (in which the LED obeys Ohm's law), holes tunnel from p-Si to n- $\mathrm{ZnO}$ through the thin $\mathrm{SiO}_{\mathrm{x}}$ layer; this mechanism is consistent with the linear I-V relation in region I. At sufficiently high voltages, it is likely that an inversion layer of holes is formed in $\mathrm{Si}$ underneath the $\mathrm{SiO}_{\mathrm{x}}$ layer. ${ }^{[34,35]}$ The rapid enhancement of EL intensity with Ga concentration can be explained by the formation of abundant $\mathrm{GaZn}-\mathrm{V}_{\mathrm{Zn}}$ pairs in the Ga-doped $\mathrm{ZnO}$ nanorods, which enable a highly efficient injection of holes into the Ga-doped nanorods where radiative recombination occurs. This mechanism is consistent with the exponential current rise in region II of the I-V curve.

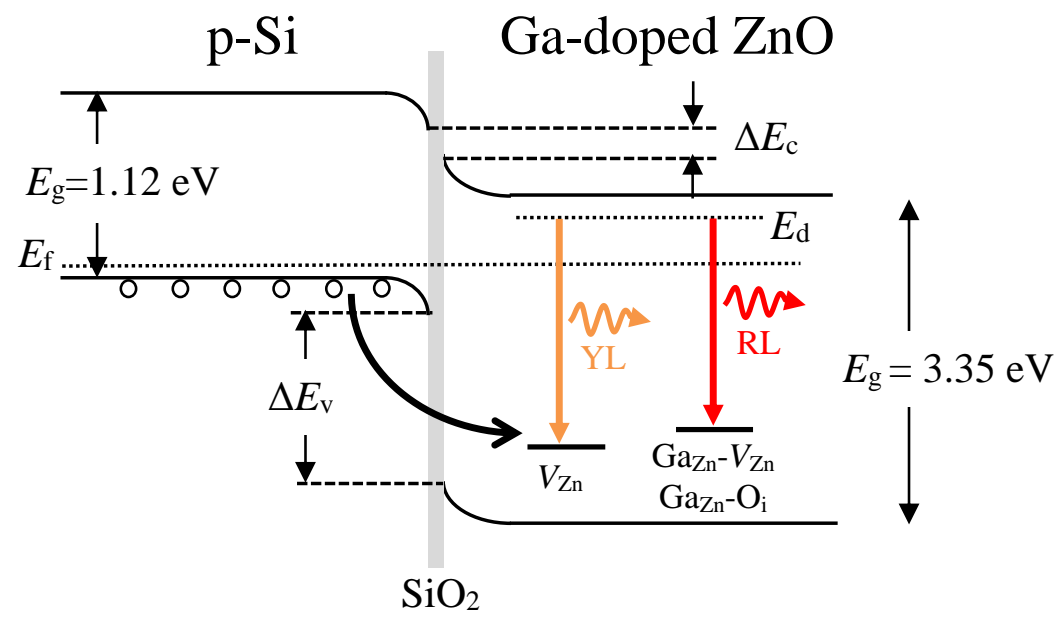

Fig. 4 Energy band diagram for p-Si/Ga-doped $\mathrm{ZnO}$ heterojunction at thermal equilibrium. The bandgap discontinuity at conduction band and valence band is therefore equal to $\Delta E_{c}=\psi_{Z n O}-$ $\psi_{S i}=0.3 \mathrm{eV}$ and $\Delta E_{v}=E_{g, Z n o}-E_{g, S i}+E_{c}=2.5 \mathrm{eV}$. Approximate positions of the defect levels are drawn based on the calculated values of $V_{\mathrm{Zn}}, \mathrm{GaZn}-\mathrm{V}_{\mathrm{Zn}}$ and $\mathrm{GaZn}-\mathrm{O}_{\mathrm{i}}$. Arrows indicate radiative recombination channels in Ga-doped $\mathrm{ZnO}$ after hole injection from $\mathrm{p}$-Si.

\section{Conclusions}

In conclusion, we have demonstrated Ga-doped $\mathrm{ZnO}$ nanorods with superior crystalline quality can be fabricated using the vapour transport method. LED devices fabricated from the p$\mathrm{Si} / \mathrm{Ga}$-doped nanorod heterojunctions exhibit a lower turn-on voltage, lower series resistance and 
enhanced EL, with the emission intensity increased by an order of magnitude than the device fabricated with undoped $\mathrm{ZnO}$ nanorods. The nanorod-based LEDs display light emission in the visible range, which are attributed to defect-related emissions in Ga-doped $\mathrm{ZnO}$. Furthermore, we show that the chromaticity of the LED devices can be tuned from yellow to red by varying the Ga content in the $\mathrm{ZnO}$ nanorods.

Supporting information. X-ray diffraction patterns of Ga-doped $\mathrm{ZnO}$ nanorods, time-resolved LA-ICP-MS depth profiles, CL spectra of undoped and 1.4 at\% Ga-doped $\mathrm{ZnO}$, determination of turn-on voltage, series- and shunt resistances, and voltage-dependent electroluminescence.

\section{Acknowledgements}

This work was supported under Australian Research Council (ARC) Discovery Project funding scheme (project number DP150103317). M. Azizar Rahman acknowledges the financial support of Australian Government through the Research Training Program Scholarship.

\section{References}

[1] C. Zhang, C. E. Marvinney, H. Y. Xu, W. Z. Liu, C. L. Wang, L. X. Zhang, J. N. Wang, J. G. Ma, and Y. C. Liu, Nanoscale 7, 1073 (2015).

[2] S. Chu, G. Wang, W. Zhou, Y. Lin, L. Chernyak, J. Zhao, J. Kong, L. Li, J. Ren, and J. Liu, Nat. Nanotechnol. 6, 506 (2011).

[3] R. Yan, D. Gargas, and P. Yang, Nat. Photonics 3, 569 (2009).

[4] Z. Mi, Y.-H. Ra, R. Wang, and R. Rashid, in Photonics Conference (IPC), 2017 IEEE (IEEE, 2017), p. 57.

[5] W. I. Park and G. C. Yi, Adv. Mater. 16, 87 (2004).

[6] M. Chen, C. Pan, T. Zhang, X. Li, R. Liang, and Z. L. Wang, ACS nano 10, 6074 (2016).

[7] P. Tao, Q. Feng, J. Jiang, H. Zhao, R. Xu, S. Liu, M. Li, J. Sun, and Z. Song, Chemical Physics Letters 522, 92 (2012). 
[8] H. Zhu, C. X. Shan, B. Yao, B. H. Li, J. Y. Zhang, Z. Z. Zhang, D. X. Zhao, D. Z. Shen, X. W. Fan, and Y. M. Lu, Adv. Mater. 21, 1613 (2009).

[9] S. W. Eaton, A. Fu, A. B. Wong, C.-Z. Ning, and P. Yang, Nat. Rev. Mater. 1, 16028 (2016).

[10] Y. Yang, Y. Li, C. Wang, C. Zhu, C. Lv, X. Ma, and D. Yang, Adv. Optical Mater. 2, 240 (2014).

[11] W. I. Park and G. C. Yi, Adv. Mat. 16, 87 (2004).

[12] F. Gao, D. Zhang, J. Wang, H. Sun, Y. Yin, Y. Sheng, S. Yan, B. Yan, C. Sui, and Y. Zheng, Appl. Phys. Lett. 108, 261103 (2016).

[13] O. Lupan, T. Pauporté, and B. Viana, J. Phys. Chem. C 114, 14781 (2010).

[14] A. Jana, S. Ghosh, P. S. Devi, N. R. Bandyopadhyay, and M. Ray, J. Mater. Chem. C 2, 9613 (2014).

[15] K. Kim, Y. Jeon, K. Cho, and S. Kim, ACS Appl. Mater. Interfaces 8, 2764 (2016).

[16] G. D. Yuan, W. J. Zhang, J. S. Jie, X. Fan, J. X. Tang, I. Shafiq, Z. Z. Ye, C. S. Lee, and S. T. Lee, Adv. Mater. 20, 168 (2008).

[17] G. C. Park, S. M. Hwang, J. H. Lim, and J. Joo, Nanoscale 6, 1840 (2014).

[18] X. Zhang, L. Li, J. Su, Y. Wang, Y. Shi, X. Ren, N. Liu, A. Zhang, J. Zhou, and Y. Gao, Laser \& Photonics Reviews 8, 429 (2014).

[19] M. Jiang, G. He, H. Chen, Z. Zhang, L. Zheng, C. Shan, D. Shen, and X. Fang, Small 13, 1604034 (2017).

[20] G.-H. He, M.-M. Jiang, L. Dong, Z.-Z. Zhang, B.-H. Li, C.-X. Shan, and D.-Z. Shen, J. Mater. Chem. C 5, 2542 (2017).

[21] C. Ton-That, M. Foley, and M. R. Phillips, Nanotechnology 19, 415606 (2008).

[22] R. Zhang, P. G. Yin, N. Wang, and L. Guo, Solid State Sci. 11, 865 (2009). 
[23] M. A. Rahman, M. T. Westerhausen, C. Nenstiel, S. Choi, A. Hoffmann, A. Gentle, M. R. Phillips, and C. Ton-That, Appl. Phys. Lett. 110, 121907 (2017).

[24] C. Ton-That, L. Weston, and M. R. Phillips, Phys. Rev. B 86, 115205, 115205 (2012).

[25] H. Matsui, H. Saeki, H. Tabata, and T. Kawai, Japanese journal of applied physics 42, $5494(2003)$.

[26] D. O. Demchenko, B. Earles, H. Y. Liu, V. Avrutin, N. Izyumskaya, U. Ozgur, and H. Morkoc, Phys. Rev. B 84, 075201, 075201 (2011).

[27] T. Cao, L. Luo, Y. Huang, B. Ye, J. She, S. Deng, J. Chen, and N. Xu, Sci. Rep. 6, 33983 (2016).

[28] Z. Shi, D. Wu, T. Xu, Y. Zhang, B. Zhang, Y. Tian, X. Li, and G. Du, J. Phys. Chem. C 120, 4504 (2016).

[29] E. F. Schubert, T. Gessmann, and J. K. Kim, Light emitting diodes (Cambridge University Press, Cambridge, U.K., 2006), p. 64.

[30] S. Chung, J.-H. Lee, J. Jeong, J.-J. Kim, and Y. Hong, Appl. Phys. Lett. 94, 168 (2009).

[31] H. Matsui, H. Saeki, H. Tabata, and T. Kawai, Jpn. J. Appl. Phys. 42, 5494 (2003).

[32] R. Anderson, IBM J. Res. Dev. 4, 283 (1960).

[33] M. J. Zhou, H. J. Zhu, Y. Jiao, Y. Y. Rao, S. Hark, Y. Liu, L. M. Peng, and Q. Li, J. Phys. Chem. C 113, 8945 (2009).

[34] S. T. Tan, X. W. Sun, J. L. Zhao, S. Iwan, Z. H. Cen, T. P. Chen, J. D. Ye, G. Q. Lo, D. L. Kwong, and K. L. Teo, Appl. Phys. Lett. 93, 013506, 013506 (2008).

[35] Y. Xu, Y. Li, H. Zhang, L. Jin, X. Fang, L. Shi, L. Xu, X. Ma, Y. Zou, and J. Yin, J. Mater. Chem. C 5, 6640 (2017). 